\title{
ON A FUNCTIONAL EQUATION
}

\section{by T. W. CHAUNDY and J. B. MCLEOD}

1. We have recently discussed in (1) the general solution of a certain functional equation arising in statistical thermodynamics, and we propose in this note to deal with another functional equation arising from the same source (2).

The problem is to obtain the most general function $f(x)$ which, for all positive integral values of $m, n$, satisfies the functional equation

where

$$
\sum_{i=1}^{m} \sum_{j=1}^{n} f\left(x_{i} y_{j}\right)=\sum_{i=1}^{m} f\left(x_{i}\right)+\sum_{j=1}^{n} f\left(y_{j}\right)
$$

$$
\sum_{i=1}^{m} x_{i}=1, \quad \sum_{j=1}^{n} y_{j}=1, \quad 0 \leqq x_{i} \leqq 1(i=1, \ldots, m), \quad 0 \leqq y_{j} \leqq 1(j=1, \ldots, n) .
$$

It is easily verified by direct substitution that a solution is

$$
f(x)=A x \log x,
$$

where $A$ is an arbitrary constant. It is the object of this note to prove that this is the only continuous solution.

It is of particular interest physically to consider infinite values of $m, n$ in (1.1). The proof given below of the uniqueness of the form of $f(x)$ does not require that (1.1) should hold for infinite values of $m, n$; it is sufficient that it should hold for all finite values. But it is readily verified that the solution

$$
f(x)=A x \log x
$$

does indeed satisfy (1.1) for infinite values of $m, n$, provided that $x_{i}, y_{j}$ are such that one side or other of the functional equation converges.

2. Let $p, q, r, s$ be any integers for which $1 \leqq r \leqq p, 1 \leqq s \leqq q$. In the functional equation (1.1), we take

and obtain

$$
\begin{aligned}
& m=p-r+1, \quad n=q-s+1, \\
& x_{i}=1 / p \quad(i=1, \ldots, p-r), \quad x_{p-r+1}=r / p, \\
& y_{j}=1 / q \quad(j=1, \ldots, q-s), \quad x_{q-s+1}=s / q,
\end{aligned}
$$

$$
\begin{aligned}
(p-r)(q-s) f\left(\frac{1}{p q}\right) & +(p-r) f\left(\frac{s}{p q}\right)+(q-s) f\left(\frac{r}{p q}\right)+f\left(\frac{r s}{p q}\right) \\
& =(p-r) f\left(\frac{1}{p}\right)+f\left(\frac{r}{p}\right)+(q-s) f\left(\frac{1}{q}\right)+f\left(\frac{s}{q}\right)
\end{aligned}
$$

Putting

$$
x f\left(\frac{1}{x}\right)=\phi(x)
$$


we then find that multiplication of (2.1) by $p q$ yields

$$
\begin{array}{r}
(p-r)(q-s) \phi(p q)+s(p-r) \phi\left(\frac{p q}{s}\right)+r(q-s) \phi\left(\frac{p q}{r}\right)+r s \phi\left(\frac{p q}{r s}\right) \\
=q(p-r) \phi(p)+q r \phi\left(\frac{p}{r}\right)+p(q-s) \phi(q)+p s \phi\left(\frac{q}{s}\right) \ldots \ldots
\end{array}
$$

If in (2.2) we take $r=s=1$, we obtain

$$
\phi(p q)=\phi(p)+\phi(q) \text {. }
$$

If in (2.2) we take only $s=1$, leaving $r$ undetermined, we obtain

$$
q(p-r) \phi(p q)+q r \phi\left(\frac{p q}{r}\right)=q(p-r) \phi(p)+q r \phi\left(\frac{p}{r}\right)+p q \phi(q),
$$

which, on substitution for $\phi(p q)$ from (2.3), yields

and so, symmetrically,

$$
\phi(p q / r)=\phi(p / r)+\phi(q),
$$

$$
\phi(p q / s)=\phi(p)+\phi(q / s) .
$$

Finally, substituting now for $\phi(p q), \phi(p q / r), \phi(p q / s)$ in (2.2), we obtain, on clearing,

i.e.

$$
\begin{aligned}
\phi(p q / r s) & =\phi(p / r)+\phi(q / s), \\
\phi(x y) & =\phi(x)+\phi(y),
\end{aligned}
$$

where $x, y \geqq 1$ and $x, y$ are rational. Continuity then extends (2.4) to all $x, y \geqq 1$.

Equation (2.4) is a well-known functional equation, of which the most general continuous solution is

$$
\phi(x)=-A \log x,
$$

where $A$ is an arbitrary constant. Referring back to the definition of $\phi(x)$, we see that

$$
f(1 / x)=\frac{1}{x} \phi(x)
$$

i.e.

$$
\begin{aligned}
f(x) & =x \phi(1 / x) \\
& =A x \log x,
\end{aligned}
$$

as required.

\section{REFERENCES}

(1) T. W. Chaundy and J. B. MCLeod, On a functional equation, Quart. J. of Math. (Oxford) (2), 9 (1958), 202-6.

(2) W. B. Brown, American J. of Phys., (to appear.)

Christ Church, Oxford

Wadham COllege, OXFord 